\title{
Turbo codes and turbo algorithms
}

\author{
Claude Berrou, Charlotte Langlais, and Yi Yu
}

\begin{abstract}
In the first part of this paper, several basic ideas that prompted the coming of turbo codes are commented on. We then present some personal points of view on the main advances obtained in past years on turbo coding and decoding such as the circular trellis termination of recursive systematic convolutional codes and double-binary turbo codes associated with Max-LogMAP decoding. A novel evaluation method, called genieinitialised iterative processing (GIIP), is introduced to assess the error performance of iterative processing. We show that using GIIP produces a result that can be viewed as a lower bound of the maximum likelihood iterative decoding and detection performance. Finally, two wireless communication systems are presented to illustrate recent applications of the turbo principle, the first one being multiple-input/multiple-output channel iterative detection and the second one multi-carrier modulation with linear precoding.
\end{abstract}

Index Terms-Turbo code, iterative decoding.

\section{INTRODUCTION}

"The oldest, shortest words - yes and no - are those which require the most thought". This pertinent observation by Pythagoras, fifth century B.C., could also be used as the motto for modern information theory. Before taking the final decision about the value of a particular bit, 0 or 1 , modern telecommunication receivers have to think a lot about it. Using probabilities and permanent, multiple dialogue between the different processors of the receiver is the key to optimal performance. Though this vision seems common sense today, it was not so obvious in the very recent past.

For instance, consider two cascaded Viterbi decoders, typically a detector followed by an error-correcting decoder. A Viterbi decoder works optimally, that is, it outputs the most likely message, taking into account the information that it has received and exploited. At the end of the 80 's, it was acknowledged that linking two locally optimal processing steps produced a globally optimal result, and this was the case for two cascaded Viterbi decoders. But this was without realizing that the first stage benefits only from part of the information available: the detector does not know that it is processing codewords, that is, bits which are not independent. Without feedback from the error-correcting decoder, the detector does not benefit from the diversity provided by the redundancy inside the codewords.

More generally, the information feeding a receiver undergoes various successive processing steps, like demodulation, detection, decoding and so on. Even if the

Manuscript received July 07, 2006 ; revised 25 August, 2006.

This paper has been approved by F. Chiaraluce.

Berrou, C. Langlais and $\mathrm{Y}$. Yu are with the GET-ENST Bretagne/PRACOM and CNRS TAMCIC, Brest, France. E-mail: Claude.Berrou@enst-bretagne.fr algorithms that are used in each of these partial steps are optimal, the accumulated result may not be optimal because the processing stream is unidirectional. And it often happens that the processor with rank $j$ can be helped by the work of the processor with rank $j+1$ or beyond, because the data that the latter possesses have not been exploited by the former. Bidirectional exchange of information between the different processors solves the problem almost ideally. It is really surprising that what today has become so obvious had been unrecognized for so long. This being said, the complexity of receivers using bi-directional message passing has noticeably increased compared to traditional structures and it is only in recent years that microelectronics has been able to cope with such complexity requirements.

The first applications of the bi-directional message passing paradigm was turbo decoding [1] and a few years later turbo equalization [2]. Subsequently, the turbo principle, as it was coined in [3], led to numerous applications involving demodulation, single and multi-user detection, synchronisation and even cryptography. The spreading of the turbo principle was also at the origin of the rediscovery of Low-Density Parity Check (LDPC) codes [4,5] which have become the matter of many studies in the information theory community.

This paper has been written to give some personal points of view on the main advances obtained these past few years on the turbo principle and the trends that are becoming apparent for the years to come. This presentation is not at all exhaustive and does not pretend to address all the ideas that have germinated in the field of turbo processing (thousands of papers have been produced on the subject). It is more like a sampling of representative questions that have been solved, more or less, or that are still under investigation. Three main sections make up the paper. The first one (chapter II) is devoted to turbo coding and decoding and presents a state-ofthe-art from the subjective point of view of the authors. In the second part (chapter III), a novel method to assess the performance of iterative processing, called genie-initialised iterative processing (GIIP), is introduced and commented on. Two recent applications of the turbo principle: multi-carrier modulation with linear precoding and multiple-in/multiple-out iterative detection are presented in the third part (chapter IV), as significant examples of the turbo principle generalisation.

\section{TURBO CODING AND DECODING}

II.1 The basic ideas that prompted the coming of turbo coding and decoding

The most powerful channel coding scheme known before 1993 was the standard concatenated code as depicted in Fig. 1, along with its decoder. We will not go into details with respect 
to the justification of this structure (see [6] for instance) but merely try to explain, with the passing of time, why this is not an optimal scheme, while it is still in use in modern systems such as DVB-T ([7]).

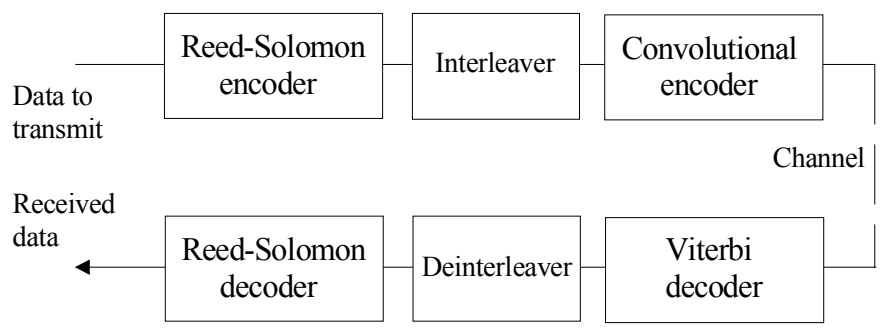

Figure 1. The standard concatenated code and its composite decoder.

The first comment that comes to mind is about the type of convolutional code used in the concatenated structure: a nonrecursive non-systematic code whereas recursive systematic convolutional (RSC) codes generally offer better performance, especially when puncturing is performed [8]. RSC codes have been brought back into favour through turbo codes mainly thanks to their ability to enable parallel concatenation, but they also have advantages with respect to performance, which is a free added bonus.

The second observation concerns the large sub-optimality of the decoder, as already discussed in the introduction. When the decoding process amounts to a single passage through the two component decoders, the inner Viterbi decoder does not benefit at all from the redundancy that the Reed-Solomon codewords contain. To make this possible, a feedback (turbo) strategy has to be carried out. But, to recall the sentence of Pythagoras at the beginning of this paper, the data to be brought back to the Viterbi decoder input must not be merely the binary decisions of the Reed-Solomon decoder. Soft values, that is, probabilities or logarithms of likelihood ratios (LLR) when working in the logarithmic domain, have to be introduced. Moreover, these probabilities or LLRs have to be constructed in such a way that correlation effects in the feedback loop are minimized. It is then imperative that the data sent back to the Viterbi decoder do not contain its own decisions. It was very easy to imagine the so-called extrinsic information which is simply obtained by subtraction when handling LLRs.

This being said, the standard concatenation depicted in Fig. 1 does not easily lend itself to turbo decoding, because the Reed-Solomon decoder is not naturally a soft-in/soft-out (SISO) decoder. Other component codes have to be considered, such as convolutional codes that were first studied in a serial concatenation to demonstrate the interest of using soft values [9]. Turbo decoding was elaborated following this serial scheme, which was later transformed into a parallel concatenation [10].

Another important observation about the code of Fig. 1 is its relative weakness with respect to random coding, which is the reference as regards error-correction coding. Of course, the deinterleaver is devised so that possible packets of errors stemming from the Viterbi decoder are spread before feeding the Reed-Solomon decoder. But because the deinterleaver is regular (linewise writing and columnwise reading), it is not able to spread consecutive packets of errors in a different way each time. This characteristic is of no importance when classical non-iterative decoding is performed, because the correlation effects that interleaving regularity may produce need bi-directional message passing between the two component decoders. In this case, the correlation effects may noticeably decrease the gain offered by iterative decoding. Non regular permutations are necessary to design good turbo codes just as, in the same way, irregular control matrices are needed to construct good LDPC codes.

\section{II.2 Main advances since 1993}

The list which is proposed and commented on here is not exhaustive. It is limited to advances that have actually been adopted in real implementations because of their practical interest.

From the coding standpoint, circular (tail-biting) termination of component codes, relatively prime permutation and the use of $m$-binary convolutional codes probably constitute the main advances.

The circular trellis termination of a convolutional code involves allowing any state as the initial state and encoding the sequence so that the final state of the encoder register will be equal to the initial state [11]. The circular termination can be used for both non-recursive and recursive codes. For the latter, leading to so-called circular recursive systematic convolutional (CRSC) codes, circular termination is possible on the condition that the message length is not a multiple of the RSC generator period. This technique is powerful though very simple, enabling block encoding for any size and any rate, without any loss in performance or spectral efficiency. Moreover and above all, because a circle has no discontinuity, circular termination does not introduce any side effects and, for this reason, is well-suited to the design of multidimensional codes.

The interleaving or permutation design for turbo coding has been the subject of many studies since 1993, as has also the design of graphs for LDPC coding, which is a comparable problem. From very empirical models, like the one used for deep space turbo coding [12], to more recent proposals, permutation has evolved towards simplicity thanks to circular trellis termination which transforms perfectly convolutional codes into block codes. In this case, the starting point for devising good permutations is the so-called relatively prime or circular permutation, first in its regular version (Fig. 2), then in versions that include some controlled disorder such as the dithered relatively prime (DRP, [13]) or the almost regular permutation (ARP, [14]) models, the latter having been adopted in several standards such as [15]. Such simple mathematical interleavers (as opposed to interleavers defined by a look-up table) are quasi-optimum with respect to the span 
(i.e. the sum of the spatial distances that separate any couple of encoded symbols, before and after the permutation), while being able to discard most possible error patterns with a rectangular shape [14].

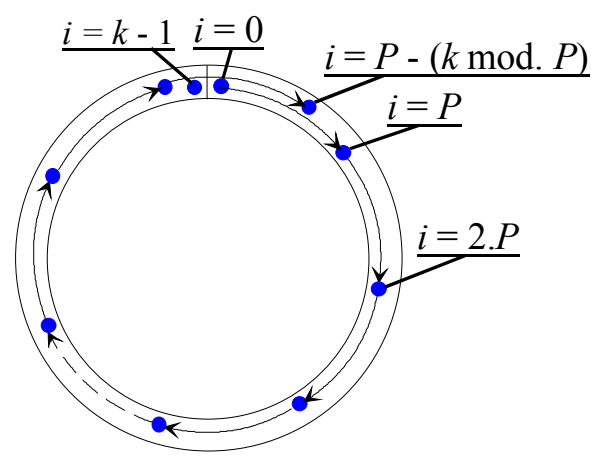

Figure 2. Regular relatively prime (or circular) permutation. The skip parameter $P$ is relatively prime with block length $k$.

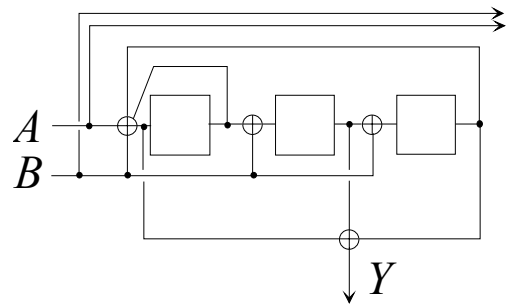

Figure 3. A double-binary recursive systematic convolutional (RSC) code with memory 3. $A$ and $B$ are the inputs, $A, B$ and $Y$ are the outputs. The natural coding rate is $2 / 3$.

In the history of information theory, non-binary coding like Reed-Solomon coding has largely proved its efficiency to lead to powerful error-correction schemes. There are at least two ways to construct a convolutional code with $m$-bit input symbols, either from the Galois field $\operatorname{GF}\left(2^{m}\right)$, or from the Cartesian product $(\mathrm{GF}(2))^{m}$. A code elaborated in $\mathrm{GF}\left(2^{m}\right)$ with code memory $v$ exhibits $2^{\mathrm{vm}}$ possible states, while the number of states of the code devised in $(\mathrm{GF}(2))^{m}$ has only $2^{v}$ states, with the same value of $v$. Fig. 3 gives an example of a doublebinary convolutional code defined in $(\mathrm{GF}(2))^{2}$. The advantages of $m$-binary turbo codes by comparison with binary turbo codes are various and have been commented on in [16].

From the decoding point of view, three major advances among many seem to stand out: the Max-log-MAP simplification of the MAP (Maximum a posteriori) algorithm [17], the introduction of the scaling factor on extrinsic information, and the possibility to implement parallelism in a turbo decoder.
When turbo decoding was elaborated to open up the way to the first integrated turbo encoder/decoder [18], the MAP algorithm was unknown to the inventor [19]. The SISO which was used at that time was the soft-output Viterbi algorithm (SOVA) inspired both by [20] and [21]. The MAP algorithm was used later only for the needs of the first publication [1], while considered as far too complex to replace the SOVA in practical implementations. Today, thanks to the work initiated in [22], simplified versions of the MAP algorithm have been adopted instead of the SOVA. The complexity of a MAP component decoder is now roughly twice that of a Viterbi decoder and, as pointed out in [23], the 8-state turbo decoder used in $3 \mathrm{G}$ mobile phone [24] represents, with 6 iterations, fewer states to process than the 256-state Viterbi decoder, which is the other decoder of the same standard. Moreover, when considering the drastic simplification of the MAP algorithm into the Max-log-MAP version, which does not require the knowledge of the noise variance, unlike the former, it is very surprising to observe no significant performance degradation, especially for $m$-binary turbo codes [16]. In fact, it is better to use the Max-log-MAP version with an appropriate scaling factor for extrinsic information (see below) than the full MAP algorithm with a poorly estimated value of the noise variance.

The scaling factor which is applied to extrinsic information before being used by either of the two decoders plays a double favourable role. First, it alleviates the correlation effects in the iterative process coming from the loops or cycles that cannot be avoided in the graph of the finite-length turbo or LDPC code. Second, the scaling factor is very favourable when using the Max-log-MAP algorithm instead of the full MAP algorithm. The simplified version, in which the maximum function substitutes for the sum, tends to overestimate the LLRs and the scaling factor helps compensate this overestimation. A proper value for the scaling factor is around 0.7 or 0.8 , except for the last iteration during which it can be 1 in order for the decoders to benefit to the full from the minimum Hamming distance of the code.

Working out parallelism in turbo decoders is a fairly recent concept $[25,14]$ motivated by the competition that the natural parallelism in LDPC decoders has initiated. Since the most of the material complexity of an iterative decoder is due to the storage requirement, especially that of extrinsic information, the architectures that allow several processors to work together in order to speed-up the decoding, without increasing the memory capacities, are not a large price to pay. Thanks to the parallelism and to the clock frequencies available today, data throughputs have reached impressive speeds, even with the field programmable gate-array (FPGA) technology. As for latencies, because the most powerful codes call for permutations or graphs that are highly irregular, their iterative decoding needs the whole received codeword in order to be started. This is the minimum delay that iterative decoding imposes and that remains too penalizing for some applications, compared with the latency of a simple Viterbi decoder, for instance. 


\section{ASSESSING ITERATIVE DECODING: THE GENIE-INITIALISED ITERATIVE PROCESSING (GIIP) METHOD}

Iterative decoding and more generally iterative processing of any concatenated functions are problems that fall into the field of non-linear dynamic systems. In view of the large number of variables to take into account, that is, in practice, hundreds or thousands of binary values combined with as many noise levels, no rigorous method seems conceivable and only partial and/or approximate tools are available for analysis or evaluation.

Two distinct types of behaviours, corresponding to small and to large signal-to-noise ratios (SNR) respectively, have to be considered in a turbo process. In the low SNR region, performance depends on many parameters, the main one most certainly being the existence of impassable theoretical limits. These limits were defined and calculated by Shannon [26] assuming a certain number of hypotheses (random coding, Gaussian channel with continuous input, infinite block size, etc.) which are more or less valid in practice. It is often difficult, even impossible, to know accurately the true limits which depend on the type of channel, the modulation scheme, the spectral efficiency, the block size and the target error rate. Therefore, it is not easy to judge whether a coding/decoding scheme is close to the optimum or largely sub-optimal. Moreover, if there is some difference between the practical performance and the supposed theoretical limit, the question arises whether to attribute it to the imperfectness of the code or to the flaws of the decoder, and the answer is not straightforward.

As for the large SNRs, the performance is mainly guided by just two parameters: the minimum Hamming distance $d_{\min }$ and the multiplicity $N\left(d_{\min }\right)$ which is the number of codewords that differ from the transmitted one by $d_{\min }$ symbols. The frame error rate (FER) that maximum likelihood (ML) decoding could achieve is then well approximated by the following formula:

$$
\mathrm{FER} \approx \frac{1}{2} N\left(d_{\min }\right) \operatorname{erfc}\left(\sqrt{R d_{\min } \frac{E_{b}}{N_{0}}}\right)
$$

where $R$ is the coding rate and $\operatorname{erfc}()$ is the complementary error function. Thus, for large SNRs and if $d_{\min }$ and $N\left(d_{\min }\right)$ are known, it is easy to make one's mind about the quality of the decoder from the real performance it achieves.

We are now going to describe a very simple method which apparently gives a tight lower bound of ML performance in the context of iteratively decodable codes. This method is just a tool to gauge the quality of coding/decoding schemes and has no application in real systems.

Let us consider an iterative error-correcting decoder which is able to receive two kinds of data: uncorrupted codewords and the same codewords but stemming from a real channel with noise and fading. Suppose that the decoder works first on the uncorrupted version of a codeword, during a certain number $n_{\mathrm{it}, 1}$ of iterations (stage 1). Of course, the decoder does not produce any errors in this ideal configuration. Then, without changing anything in the state of the decoder, that is, without altering the pieces of extrinsic information that have stabilized after $n_{\mathrm{it}, 1}$ iterations, the corresponding corrupted codeword comes to replace the true one at the decoder input. The decoder works again during $n_{\mathrm{it}, 2}$ iterations (stage 2).

Amazingly (at least for the person who conducted this experiment for the first time), the error rate is no longer 0 at the end of the process. Though extrinsic pieces of information have reached their full level at the end of the first stage and thus can act as strong correct a priori inputs to the component decoders, this is not sufficient to prevent the decoder from drifting to a bad decision during the second stage in the case of strong corruption. In fact, stage 1 constitutes a perfect initialisation process towards the classical iterative decoding performed in stage 2. This perfect initialisation actually appears to give the decoding the ability to achieve performance close to that of ML decoding. More precisely, this technique that we have called genie-initialised iterative processing (GIIP), produces a result that can be considered as a lower bound of the ML decoding performance, according to the following rationale.

As explained in [27], turbo and LDPC decoding, when referred to as Pearl's belief propagation (BP) algorithm, are optimal when the graph of the coding structure is free of any cycle. If cycles exist, and there are cycles in any finite-length turbo or LDPC code, the iterative decoding suffers from correlation effects in the message passing between component decoders. So let us assume that the difference in performance between ML decoding and turbo or LDPC decoding comes solely from correlation effects. These may be either favourable or unfavourable, the average leading to a worsening of the performance. The favourable correlation effects concern extrinsic pieces of information that are correct, while unfavourable effects are related to erroneous pieces of information. When stage 1 is performed in the GIIP process, all correlation effects are favourable because all data are correct. Then, when stage 2 begins with strong (and overestimated owing to correlation) correct a priori inputs to the component decoders, all the correlation effects are still exclusively favourable and the result of the GIIP decoding is better than that given by decoding without correlation, that is, ML decoding according to [27].

To illustrate the GIIP assessment technique, we consider the example of the double-binary turbo code used in the DVBRCS [28] and WiMax [15] with $k=1504$ (188 bytes), $R=1 / 2$ and QPSK modulation on a Gaussian channel. The component decoding algorithm is the Max-Log-MAP algorithm and 30 iterations are performed for both the classical and the GIIP simulations $\left(n_{\mathrm{it}, 1}=10, n_{\mathrm{it}, 2}=30\right)$. Figure 4 gives the result of these simulations in terms of FER, along with the theoretical limit obtained with the sphere-packing approach. 


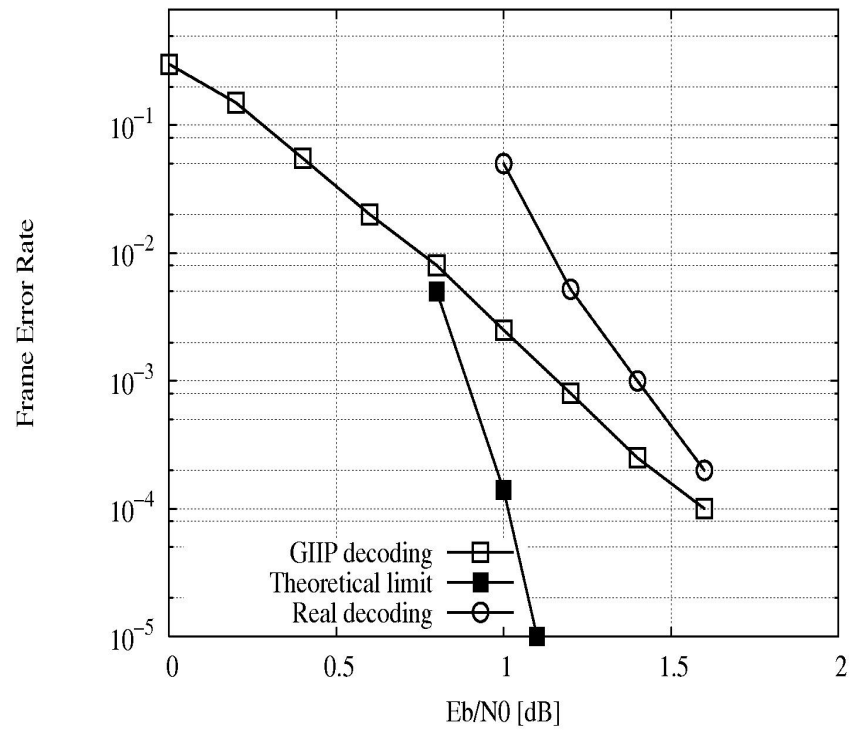

Figure 4. Comparison of real and GIIP decoding performance of a double-binary turbo code with $k=1504$ information bits, $R=1 / 2$ and QPSK modulation on a Gaussian channel.

Several observations can be made from the curves of Fig. 4. First, the GIIP decoding result seems compatible with the sphere-packing limit whose upper part (for large FER) is not represented for lack of accuracy. If the minimum Hamming distance of the code considered were much larger than the real one, we could imagine that the GIIP decoding curve would fall steeply to values close to the theoretical limit curve. Second, for large SNR, real decoding offers performance that asymptotically catches up the GIIP performance, which we recall is considered as a lower bound of ML decoding. Another observation concerns the small gap (less than $0.6 \mathrm{~dB}$ for practical values of FER) lying between the real and genieinitialised simulation results, clearly indicating that the correlation effects are not of very great importance in turbo decoding, at least for this particular case of the double-binary turbo code, with $k=1504$ and $R=1 / 2$.

The GIIP technique will be used in chapter IV to assess the performance of two other systems with iterative receivers. Generally speaking, this new method for the evaluation of iteratively decodable schemes could bring precious information about:

- the potential quality of a transmitter design, regardless of the receiver sub-optimality,

- the quality or the weakness of an iterative receiver, compared to ML processing,

- the theoretical limits "in practice". If we assume that the ML decoding of a random-like code, such as turbo or LDPC, is able to reach the capacity limits (at least for medium error rates), then the GIIP technique could provide lower bounds of these limits in an experimental way. This can be done without any mathematical development which generally turns out to be very intricate for real situations, especially for short or medium block sizes. On the contrary, the GIIP method does not require any assumptions to be made.

\section{THE TURBO PRINCIPLE COMES INTO WIDE USE}

Information feeding a receiver undergoes several successive processing steps. These steps can be roughly classified into three categories: detection, estimation and decoding. In conventional representation, only unidirectional processing is performed. Detection and estimation ignore the decoding step. As largely commented on in the previous sections, this leads to a sub-optimal process. In the sequel we focus on the detection step in order to present two significant applications of the turbo principle other than mere channel decoding.

\section{IV.1 MIMO iterative detection}

Since the next-generation wireless communications are driving the demand for increased system capacity, data rates and multimedia services, the multiple-in/multiple-out (MIMO) channel has recently emerged as one of the most significant technical breakthroughs in modern digital communications. Perhaps even more surprising is that just a few years after its invention, the technology seems poised to penetrate largescale standards-driven commercial wireless products and networks such as broadband wireless access systems, wireless LANs (e.g. WiMax), third-generation (3G) networks and beyond [29]. A key idea in MIMO systems is the space-time signal processing in which the time is complemented with the spatial dimension inherent in the use of multiple spatially distributed antennas. Several coding techniques have been proposed with multiple transmit and/or receive antennas in various fading channels to achieve transmit diversity and spatial diversity such as space-time block codes (STBC) [30] and Bell Laboratories layered space-time (BLAST) codes [31]. To obtain the additional diversity gain, numerical methods were developed for MIMO systems. These could be broadly categorized into two techniques: one is the open-loop scheme such as the optimum constellation rotation [32] and the other is the closed-loop scheme that is based on channel state information (CSI) sent from the receiver to the transmitter through an independent feedback channel.

In order to exploit the available capacity of MIMO systems, recent attention has turned to iterative detection and decoding at the receiver side. It is well known that bit-interleaved coded modulation (BICM) is a power and bandwidth efficient coded modulation scheme. Inspired by turbo decoding, iterative decoding between a demapper and a decoder can be applied to BICM. It is demonstrated that BICM with iterative decoding (BICM-ID) outperforms trellis-coded modulation (TCM) in different channel models when the constellation labelling is properly designed [33]. Motivated by the above advantages, this technique has been extended to the MIMO channel in order to achieve a significant coding gain. Two concatenation structures were designed for multiple transmit and receive antenna systems. In [34], Tonello proposed BICM-ID combined with a vertical-BLAST (V-BLAST) scheme, which can be termed as space-time bit-interleaved coded modulation with iterative decoding (ST-BICM-ID). The other efficient structure based on the concatenation between BICM-ID and a STBC was called bit-interleaved space-time coded modulation 
with iterative decoding (BI-STCM-ID) [35]. Since V-BLAST offers high spectral efficiencies by dividing the incoming data into multiple sub-streams and sending each sub-stream to a dedicated antenna, ST-BICM-ID can achieve a desired high transmission rate at the expense of loosing some degrees of diversity and increasing the decoding complexity. For BISTCM-ID, STBC provides the highest diversity advantage for MIMO systems with a low complexity decoding algorithm. Although its association with a powerful channel code can improve both diversity gain and coding gains, this scheme suffers from the transmission rate. The transmitters of these two iteratively decoded MIMO systems are depicted in Fig. 5 (a) and (b).

The main difference between these two transmitters is the selected space-time mapping scheme before the transmit antennas. As shown in Fig.5 (a), the serial to parallel (S/P) component is used to spatially distribute the modulated signals to multiple antennas. As for the BI-STCM-ID transmitter, the STBC encoder maps the same signals in a permuted order to transmitting antennas during different time durations. At the output of the MIMO channel we receive the observation $\mathbf{Y}=\mathbf{H X}+\mathbf{N}$, where $\mathbf{X}$ is a vector of the transmitted signals that are made up of the modulated symbols, $\mathbf{H}$ is an equivalent channel matrix in which the channel coefficients are modelled as independent and identically distributed (i.i.d) complex Gaussian random variables with zero mean and unit variance, and $\mathbf{N}$ is a zero-mean complex Gaussian noise vector with variance $\sigma_{n}^{2}$.

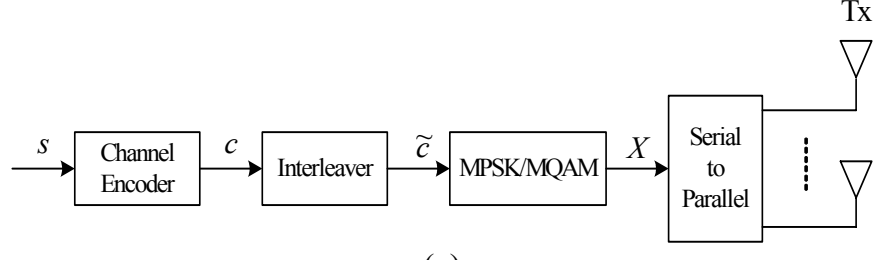

(a)

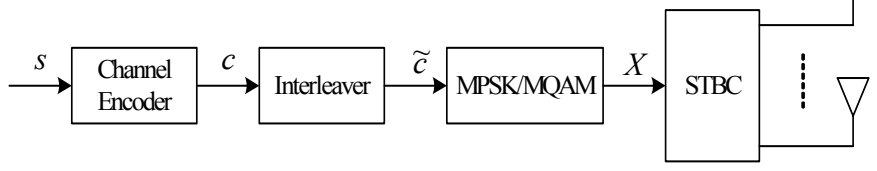

(b)

Figure 5. Two transmitters of the turbo-MIMO family, (a) ST-BICM-ID and (b) BI-STCM-ID.

The receiver is assumed to have the perfect channel knowledge and consists of a MIMO detector and an $a$ posteriori probability (APP) decoder. Both are SISO devices and exchange iteratively soft information about the encoded bits according to the turbo principle[3]. The general iterative receiver model for both systems is shown in Fig. 6.

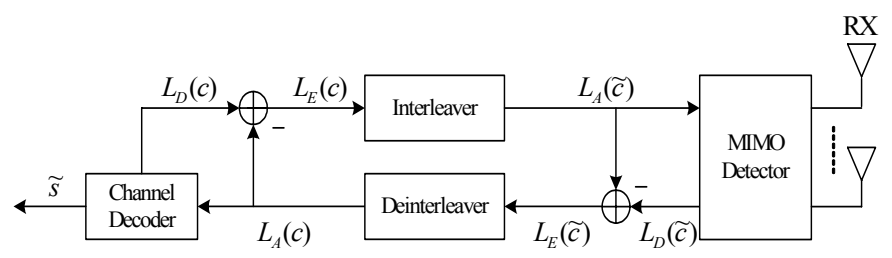

Figure 6. The general iterative receiver model for turbo-MIMO systems.

Consider the ML receiver for which the MIMO demapping algorithm exploits the equivalent channel matrix $\mathbf{H}$. The $\log$ likelihood ratio (LLR) of the bit $c_{i}, i=1, \ldots, N_{t} \cdot m$ (where $N_{t}$ and $m$ represent the number of transmit antennas and the number of bits included in one constellation point, respectively), conditioned on $\mathbf{Y}$ and $\mathbf{H}$, is

$L_{D}\left(c_{i} \mid \mathbf{Y}, \mathbf{H}\right)=L_{A}\left(c_{i}\right)+\ln \underbrace{\left[\sum_{\mathbf{X} \in \Psi_{1}^{\mathrm{i}}} P(\mathbf{Y} \mid \mathbf{X}, \mathbf{H}) \cdot \exp \left(\sum_{j \neq i} \frac{1}{2} \mathrm{c}_{j} L_{A, j}\right)\right.}_{L_{E}\left(c_{i}\right)} \underbrace{}_{\left.j(\mathbf{Y} \mid \mathbf{X}, \mathbf{H}) \cdot \exp \left(\sum_{j \neq i} \frac{1}{2} \mathrm{c}_{j} L_{A, j}\right)\right]}$

where $\Psi_{1}^{\mathrm{i}}\left(\right.$ or $\left.\Psi_{0}^{\mathrm{i}}\right)$ is a set of $2^{N_{t} \cdot m}$ bit vectors $\mathbf{X}$ with the

binary bit 1 (or 0 ) at labelling position $i, \mathrm{c}_{j}$ is the $j$ th bit in each vector associated with $\mathbf{X}, L_{A, j}$ denotes the $j$ th a priori

information $L_{A}$ and $P(\mathbf{Y} \mid \mathbf{X}, \mathbf{H})$ is the conditioned

probability density function (pdf), which is given by:

$$
P(\mathbf{Y} \mid \mathbf{X}, \mathbf{H})=\exp \left(-\frac{\|\mathbf{Y}-\mathbf{H} \cdot \mathbf{X}\|^{2}}{2 \sigma_{n}^{2}}\right)
$$

Further simplifications are possible by using the Max-Log approximation which transforms the LLR-valued extrinsic information $L_{E}\left(c_{i}\right)$ into:

$$
\begin{array}{r}
L_{E}\left(c_{i}\right)=\max _{\mathbf{X} \in \Psi_{1}^{i}}\left(-\frac{\|\mathbf{Y}-\mathbf{H} \cdot \mathbf{X}\|^{2}}{2 \sigma_{n}^{2}}+\frac{1}{2} \sum_{j \neq i}\left(\mathrm{c}_{j} \cdot L_{A, j}\right)\right) \\
-\max _{\mathbf{X} \in \psi_{0}^{i}}\left(-\frac{\|\mathbf{Y}-\mathbf{H} \cdot \mathbf{X}\|^{2}}{2 \sigma_{n}^{2}}+\frac{1}{2} \sum_{j \neq i}\left(\mathrm{c}_{j} \cdot L_{A, j}\right)\right)
\end{array}
$$

It is worth pointing out that the number $N_{b}$ of computations required to obtain the LLR for each bit in ST-BICM-ID systems grows exponentially with the constellation size $2^{m}$ $\left(N_{b}=2^{m \times N_{t}}\right)$. On the other hand, for BI-STCM-ID systems, $N_{b}$ grows linearly $\left(N_{b}=2^{m}\right)$ instead of exponentially. For example, if 16-QAM is adopted for the two systems with two 
transmit antennas, 256 comparisons have to be performed for the ST-BICM-ID system when the ML rule is considered, while only 16 comparisons are needed for the BI-STCM-ID system. This significant hardware reduction is very attractive for VLSI implementations. To alleviate the drawback of the receiver complexity in ST-BICM-ID systems, several efficient algorithms such as sphere decoding [36] can be used to further reduce decoding complexity.

In the following, we apply the GIIP technique, as introduced in chapter III, to a particular turbo-MIMO system in uncorrelated block fading channels. The (2 Tx, $1 \mathrm{Rx})$ Alamouti code and a $(37,21)$ CRSC code of $1 / 2$ coding rate are considered as the inner and outer code, respectively. Moreover, we use an anti-Gray mapping scheme for the QPSK constellation. $n_{\mathrm{it}, 1}=10, n_{\mathrm{it}, 2}=8$ for GIIP decoding, and 8 iterations for real decoding are considered in this system. As Fig. 7 shows, there is almost no difference between the GIIP and real decoding. This is mainly explained by the property of the detector which delivers symbols that are not much correlated. Actually the Alamouti code links the data in independent pairs and the correlation effects are limited within these couples of symbols. Therefore, the classical iterative processing without GIIP is already close to ML processing. However this experiment was useful to demonstrate that the GIIP technique provides a tight lower bound to ML performance.

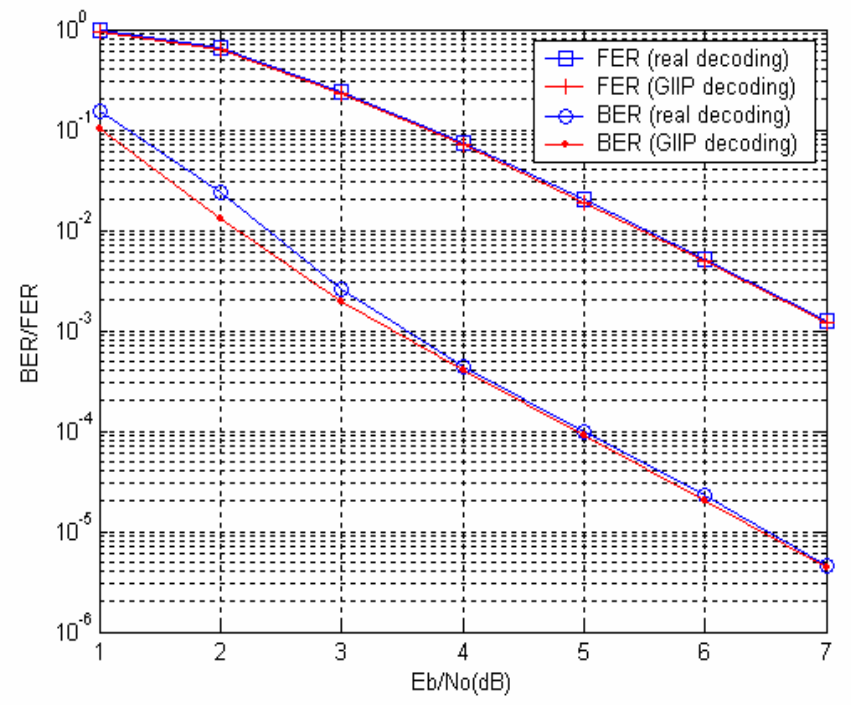

Figure 7. Comparison between GIIP and real decoding for the turboMIMO system.

\section{IV.2 Linear Precoding}

In this section we concentrate on the application of the turbo principle to a specific diversity technique called linear precoding. Unfortunately, this term can be related to several very different communication techniques. So before delving into the details of the turbo receiver, we briefly review the principle of this diversity technique.

\section{Linear Precoding technique as a diversity technique}

Diversity techniques are efficient for improving the performance, in terms of error rates, of communication systems on the Rayleigh fading channel. On such a channel, information theory tells us that the Bit Error Probability decreases only linearly with the Signal to Noise Ratio (SNR). Suppose that one bit is sent through the channel. If at that time, the fading is deep, the received signal power is so weak that no reliable detection can be carried out. Now, the bit is sent twice at two different time slots. If these two time slots correspond to two independent channel realizations, the probability that the two fadings are deep is lower than the probability that only one fading is deep. The detection of the transmitted bit involves the combination of the two received signals such as the selection of the strongest signal or the linear combination of both. The BER performance of this system based on repetition coding is improved compared with the conventional system. In this example, the channel variations in the time domain provide time diversity that can be exploited by a properly designed transmitter/receiver couple. In the same way, in the case of a multi-path propagation channel, frequency diversity techniques can be carried out to take advantage of the channel variations in the frequency domain due to frequency selectivity. The final BER performance depends on the degree of diversity provided by the channel, which can be infinite in the case of white Rayleigh fading, and the degree of diversity that can be exploited by the transmitter/receiver couple.

In the previous example, the technique of repetition coding was carried out to exploit the time diversity provided by the channel. However, more rate efficient techniques exist such as linear precoding. Chronologically, linear precoding was first applied to a multi-carrier transmission called Orthogonal Frequency Division Multiplex - Code Division Multiplexing (OFDM-CDM) [37]. This technique was presented as a generalised case of the Multi Carrier - Code Division Multiple Access (MC-CDMA) system that combines advantages from OFDM and CDMA. Before the Inverse Fast Fourier Transform, the complex symbols are spread according to a user-specific spreading code. The orthogonality of the spreading codes enables the efficient separation of the users' signals at the receiver. Unfortunately, if the transmitted signal experiences channel fading, orthogonality is lost and Multiple Access Interference (MAI) appears, degrading the BER performance. Nevertheless, the spreading code, acting as a repetition code, can be used to offer frequency diversity. Now, if all spreading codes are allocated to one single user, no loss in data rate is experienced and the system retains its capacity to exploit frequency diversity. Obviously, MAI is converted into Self Interference (SI). Depending on the designed detector at the receiver part, the diversity gain is not able to compensate for the BER degradation due to SI. This diversity technique has also been explored independently from another point of view by [38]. It was then generalised by [39] under the name of Linear Precoding (LP). 
The baseband equivalent model of an LP-OFDM system is depicted in Fig. 8. Data are encoded using a BICM scheme with a rate $R$ convolutional encoder. Coded bits are mapped onto complex symbols $\left(s_{i}\right)$ chosen in the constellation $\chi$ (BPSK, QPSK, $2^{q}$-QAM...) using Gray mapping. Linear precoding involves spreading each information symbol over several time intervals or subcarriers. Each vector of symbols $\mathbf{s}$ $=\left(s_{0}, \ldots, s_{M-1}\right)$ of size $M$ is multiplied by an $M \mathrm{x} M$ unitary precoding matrix $\mathbf{D}_{M}=\left(d_{i, j}\right), i, j=1, . ., M$. This operation can be viewed as a block coding of size $M$ with rate equal to 1 . The OFDM channel includes a symbol interleaver $\Pi_{2}$, the OFDM modulation/demodulation and the multipath frequency-selective channel. OFDM operations such as the cyclic prefix insertion prevent Inter Symbol Interference (ISI) and Inter Carrier Interference (ICI). As a consequence, the relationship between the transmit symbols $\mathbf{s}$ and the output $\mathbf{r}$ of the OFDM channel can be written as

$$
\mathbf{r}=\mathbf{H D}_{M} \mathbf{s}+\mathbf{w}
$$

where $\mathbf{H}=\operatorname{diag}\left(h_{0}, \ldots, h_{M-1}\right)$ is the $M \times \mathbf{x} M$ diagonal complex matrix, bearing the frequency channel gains on its diagonal, and $\mathbf{w}=\left(w_{0}, \ldots, w_{M-1}\right)$ an additive white Gaussian noise such that $\mathrm{E}\left(\mathbf{w w} \mathbf{H}^{\mathrm{H}}\right)=\sigma_{w}^{2} \mathbf{I}_{M}$.

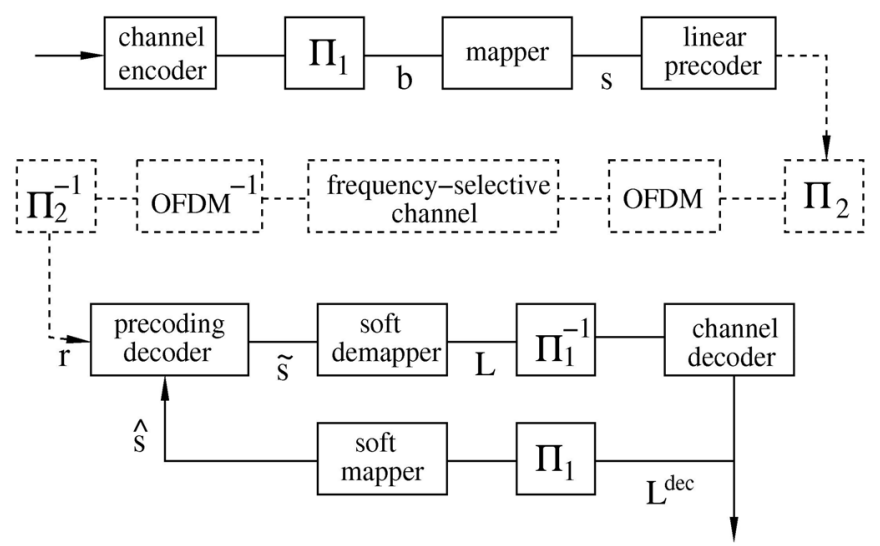

Figure 8. Transmission scheme for linear precoding (LP) communication systems.

The interleaver $\Pi_{2}$ maps precoded symbols onto different sub-carriers and OFDM symbols. Thus, the LP scheme can take advantage of both time and frequency diversity. Each information complex symbol sees different $M$ frequency channel gains. Assuming perfect frequency interleaving, the frequency channel gains can be modelled as Rayleigh distributed random variables. So, the maximal diversity order $D$ that LP could provide is intuitively equal to $M$. A lower bound of the coded LP-OFDM system could coincide with the union bound of a coded system over $M$ diversity branches assuming a Maximum Ratio Combining (MRC) receiver. At high SNR this bound is given by

$$
P(e) \leq \sum_{d=d_{\min }}^{+\infty} A_{d}^{(b)}\left(\begin{array}{c}
2 M d-1 \\
M d
\end{array}\right)\left(\frac{1}{4 R E_{b} / N_{0}}\right)^{M d}
$$

where the couples $\left(A_{d}^{(b)}, d \in\left[d_{\min }, \ldots \infty\right]\right)$ are the components of the distance spectrum of the channel code. Only the computation of the Maximum Likelihood decoding bound could acknowledge this intuition. This bound could particularly help us in the design of the LP matrix and provide an estimation of the achievable diversity order of the coded LP-OFDM system. Unfortunately, the mathematical development of this bound does not exist. At the end of this section, we will see how the GIIP method can be applied to solve this problem.

The achievement of the potential diversity order is also contingent upon a properly designed receiver. If we consider channel coding and LP as separate tasks, the optimal detector for the LP task is based on ML decoding. This decoding involves an exhaustive search for the most reliable complex symbol vector among the set of all possible complex symbol vectors. Nevertheless the cardinality of this set, equal to $2^{q M}$, leads to prohibitive complexity for $q>2$. Moreover the performance depends on the matrix design. In [39], specific matrices for $M=2$ and $M=4$ have been designed achieving the maximal diversity order.

To reduce the receiver complexity, Minimum Mean Square Error (MMSE) and Zero Forcing (ZF) detectors inspired by single user linear detectors can be implemented. However they have been rapidly replaced by more powerful detectors such as Soft Interference Cancellers (SIC) because of their low performance, far from the performance of the $M$-branch diversity system.

Considering channel coding and LP as separate tasks is suboptimal from the information theory point of view. The intuitive understanding of this sub-optimality relies on the following observation. Since the LP detector has no knowledge of the channel code constraints and is performed at the receiver front end, it cannot take advantage of the channel decoder work. The sub-optimality of this receiver design exists even if matrices optimised for maximal diversity order associated with ML detector are used [40]. In fact, the accumulated result of optimal partial operations may not be optimal because the processing stream is unidirectional.

\section{Turbo detection for LP systems}

The optimal receiver in terms of BER performance is obviously the ML receiver operating on the joint channel coder, interleaving and LP process. However, this receiver is completely intractable, not only for hardware implementation but also for performance evaluation through Monte-Carlo simulations.

So since the sub-optimality comes from the unidirectional stream, the turbo principle will help us to overcome the intractability of the joint ML receiver. This principle is carried out in introducing a bi-directional stream between the LP detector and the channel decoder. 
As the LP detector does not take into account any soft $a$ priori information, a new detector has to be designed. Let us have a look on equation (4) and consider $\mathbf{H D}_{M}$ as an equivalent channel matrix. Consequently the LP system equation is a more general system equation since it can represent any system with interference such as ISI, MAI, and Spatial Interference in the case of MIMO channels.

The first solution based on the turbo principle was initially proposed for the ISI problem in the context of frequency selective channels [2]. It involves a SOVA detector taking into account a priori information unlike the ML detector. This SOVA was rapidly replaced by a MAP detector. Another solution based on the MMSE criterion with a priori information has already been proved to be competitive with the MAP turbo-equaliser in the context of frequency-selective channels [41], [42]. In the sequel we focus on the MMSE detector with a priori information applied to the LP scheme.

Fig. 9 depicts the MMSE turbo-detector. The detector is constrained to a classical Interference Canceller (IC) structure. Thanks to the unitary property of the LP matrix and to the diagonal property of the channel matrix $\mathbf{H}$, the equalisation matrix $\mathbf{P}$ is also diagonal $\mathbf{P}=\operatorname{diag}\left(p_{0}, \ldots, p_{M-1}\right)$. Let's define $\mathbf{G}=\mathbf{D}_{M}^{-1} \mathbf{P H D} \mathbf{D}_{M}=\left(g_{i, j}\right)$. The output of the MMSE detector $\widetilde{S}_{i}$ is given by:

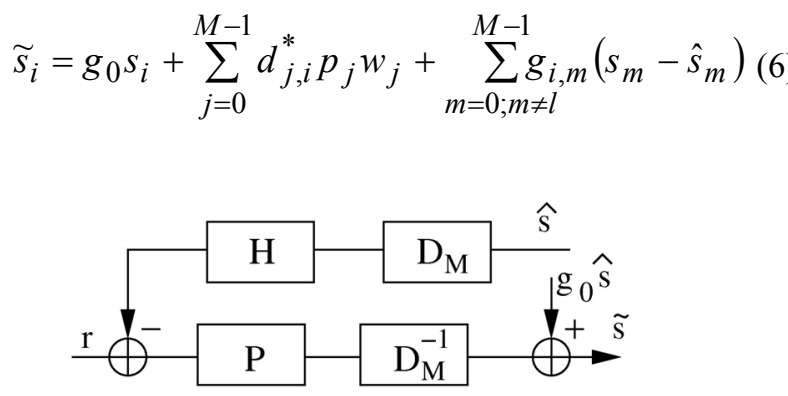

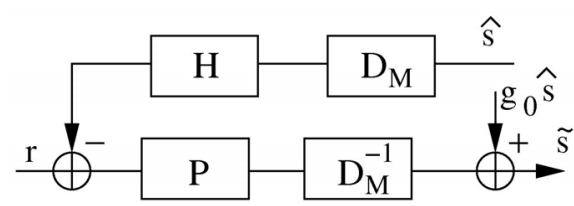

Figure 9. LP detector with a priori information.

Deriving the MMSE criterion with a priori information leads to minimise $\mathrm{E}\left[\left|\tilde{s}_{i}-s_{i}\right|^{2} \mid \hat{\mathbf{s}}\right]$. The equalisation coefficients can be written as in [43]:

$$
p_{i}=\lambda \frac{h_{i}^{*}}{\left(\sigma_{s}^{2}-\sigma_{\hat{s}}^{2}\right)\left|h_{i}\right|^{2}+\sigma_{w}^{2}}
$$

where $\lambda=\frac{\sigma_{s}^{2}}{1+\beta \sigma_{\hat{s}}^{2}}$ and $\beta=\frac{1}{M} \sum_{m=0}^{M-1} \frac{\left|h_{m}\right|^{2}}{\left(\sigma_{s}^{2}-\sigma_{\hat{s}}^{2}\right)\left|h_{m}\right|^{2}+\sigma_{w}^{2}}$.

Equation (7) provides an easy way to evaluate the equalisation coefficients, but it requires the knowledge of $\sigma_{\hat{s}}^{2}$. This variance is estimated over a decoded block after the soft mapper for each iteration using the following approximation [41]:

$$
\sigma_{\hat{s}}^{2}=\mathrm{E}\left\{\left.\hat{s}_{i}\right|^{2}\right\} \approx \frac{1}{N} \sum_{n=0}^{N-1}\left|\hat{s}_{n}\right|^{2}
$$

where $N$ is the block size. In the previous notations, the index $l$ of the current iteration has been omitted. Actually as $\hat{s}_{n}$ and $\sigma_{\hat{s}}^{2}$ are updated every iteration, the equalisation matrix $\mathbf{P}^{(l)}$ is computed once per iteration in the case of a slow fading channel (H constant over one block). The detector complexity is consequently reduced.

Two particular cases can be emphasized: the first iteration, where no a priori information is available, and the genie aided case, where $\hat{\mathbf{s}}$ is equal to $\mathbf{s}$. At the first iteration, as $\sigma_{\hat{s}}^{2}$ is equal to zero, it results in a classical MMSE equalization without interference cancellation. In the genie aided case, the equalization coefficients become:

$$
p_{i}=\frac{\lambda}{\sigma_{w}^{2}} h_{i}^{*}
$$

corresponding to the coefficients of the channel matched filter. The new expression for the estimated symbols is then:

$$
\tilde{s}_{i} \propto s_{i} \frac{1}{M} \sum_{j=0}^{M-1}\left|h_{j}\right|^{2}+\sum_{m=0}^{M-1} d_{i, m}^{*} h_{m}^{*} w_{m}
$$

As the noise term is a linear combination of independent Gaussian noise realizations, it keeps its Gaussian distribution whatever the LP matrix type. Consequently the output of the genie aided detector does not depend on the LP matrix type. The derivation of the union bound of the genie aided scheme is performed in the same way as for the coded system over $M$ diversity branches assuming a MRC receiver. This results in equation (5) [40]. Therefore if the iterative process converges towards its genie aided scheme, the system is able to exploit the maximum diversity order $M$, whatever the LP matrix type. In practice, the genie aided case does not occur since this assumes noise-free symbol estimates. Nevertheless, at high SNR and for a sufficiently large number of iterations, the iterative process has to converge towards it. The genie aided performance is consequently a lower bound for the iterative process.

Except for the genie aided case, the coefficients of the LP detector depend on the matrix type. Consequently, at the first iterations the performance of the iterative process is driven by the local performance of the LP detector and the channel decoder. In particular, the position of the trigger point can be shifted towards higher SNR when using a non optimal matrix according to the ML criterion. 
So far, the genie aided bound was the only means to assess the performance of turbo-detectors or turbo-equalizers. We propose to apply the GIIP method to the particular case of the turbo-detector for the LP scheme.

\section{Application of the GIIP technique}

The channel code considered here is a simple convolutional code and the channel decoder processes only the LLRs derived from the detected symbols supplied by the LP detector. The GIIP assessment technique as described in section III is applied to the turbo-detector. During stage 1, uncorrupted channel complex symbols are provided to the LP detector. Moreover, the LP detector benefits from the channel decoder output through the soft complex mapper. During stage 2 , the LP detector receives noisy channel complex symbols and the turbo-detector works again during $n_{i t, 2}$ iterations.

The LP scheme is performed for two kinds of matrices of size 4: Hadamard and complex matrices as defined in [44]. The Hadamard matrices are recursively defined for each $M$ power of two by:

$$
\mathbf{D}_{M}=\frac{1}{\sqrt{2}}\left(\begin{array}{cc}
\mathbf{D}_{M / 2} & \mathbf{D}_{M / 2} \\
\mathbf{D}_{M / 2} & -\mathbf{D}_{M / 2}
\end{array}\right) \quad \text { with } \quad \mathbf{D}_{1}=1
$$

The complex matrix has been devised to be optimal under the ML criterion for the LP detector alone. The matrix of size $4 \times 4$ is given by

$$
\mathbf{D}_{4}=\left(\begin{array}{cccc} 
& \mathrm{e}^{j \frac{\pi}{8}} & \mathrm{e}^{j \frac{2 \pi}{8}} & \mathrm{e}^{j \frac{3 \pi}{8}} \\
1 & \mathrm{e}^{j \frac{5 \pi}{8}} & \mathrm{e}^{j \frac{10 \pi}{8}} & \mathrm{e}^{j \frac{15 \pi}{8}} \\
1 & \mathrm{e}^{j \frac{9 \pi}{8}} & \mathrm{e}^{j \frac{18 \pi}{8}} & \mathrm{e}^{j \frac{27 \pi}{8}} \\
1 & \mathrm{e}^{j \frac{13 \pi}{8}} & \mathrm{e}^{j \frac{26 \pi}{8}} & \mathrm{e}^{j \frac{39 \pi}{8}}
\end{array}\right)
$$

The performance of the turbo-detector for the LP scheme regarding the BER is given for the 16-QAM modulation and a CRSC code with constraint length $K=7$. A puncturing pattern is applied to obtain a rate equal to $2 / 3$. The channel decoder uses the Max-Log MAP algorithm and the turbo-detector performs 8 iterations. These parameters for the channel code and modulation have been chosen in order to obtain different simulation results for the Hadamard and the complex matrices. The combination of a high rate code and high order modulation is less favourable to the Hadamard matrix [43].

With perfect time and frequency interleaving, the OFDM modulation, the frequency selective channel and the OFDM demodulation can be represented by the flat fading Rayleigh channel. Fig. 10 and 11 present the performance results of the turbo-detector over this channel, for the Hadamard matrix and the complex matrix respectively. The convergence is slightly faster with the complex matrix than with the Hadamard one, as expected. Moreover, the LP scheme with the Hadamard matrix exhibits a flaw of convergence. The genie and GIIP performance is not reached. For both cases, the performance of the turbo-detector approaches the GIIP curve without crossing it.

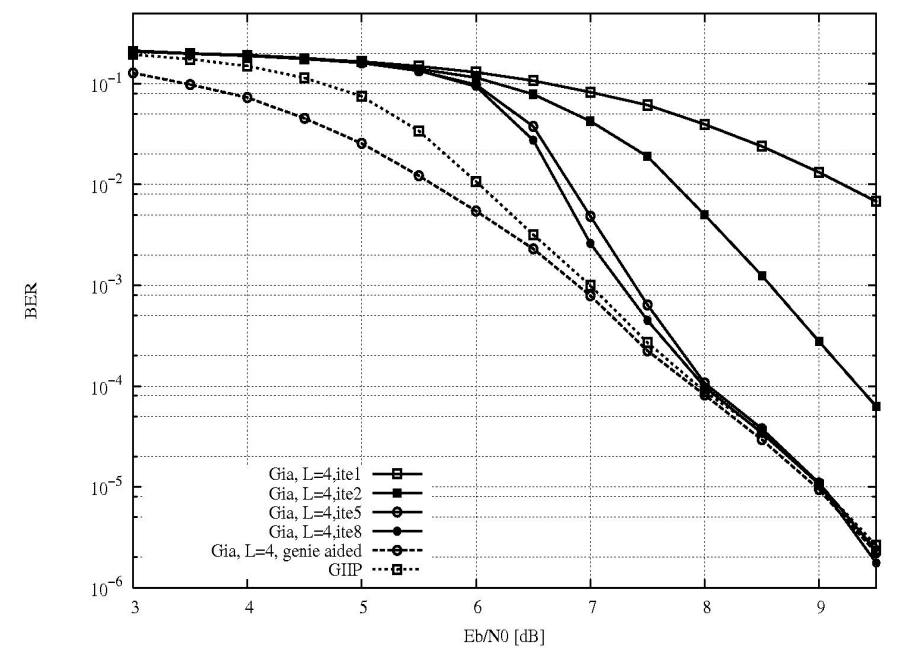

Figure 10. BER results of the turbo-detector for the LP complex matrix with 512 information bytes.

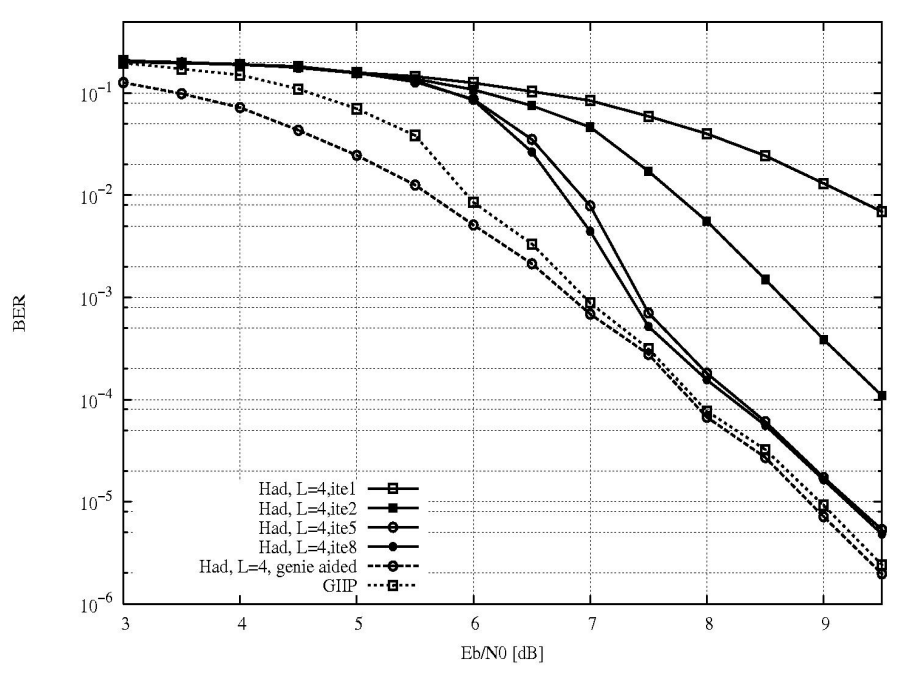

Figure 11. BER results of the turbo-detector for the LP Hadamard matrix with 512 information bytes.

The genie aided performance and the GIIP performance merge at high SNR. The difference observed at low SNR can be intuitively explained as follows. Consider that the ML decoding corresponds to a perfect bi-directional exchange (without correlation) between the LP detector and the channel decoder. At low SNR, the information provided by the channel decoder to the LP detector cannot be perfect because of the additive noise. Therefore the ML decoding is not as efficient as the genie aided receiver could let us suppose. If we consider the GIIP performance as a lower bound for the ML decoding performance, this explains the difference at low SNR. On the other hand, at high SNR, the assumption of noise-free symbol estimates becomes realistic and the performance curves merge. As well as the genie aided performance, the GIIP performance for the Hadamard and the complex matrices match perfectly. 


\section{CONCLUSION}

With the development of turbo and LDPC codes and the extension of the turbo principle, the performance of communication systems, as sophisticated as they may be, can be obtained very close to the optimum. This paper was organized as a kind of journey beginning in the early 90's and highlighting the key features of this new way of thinking information processing in receivers. Two examples of turbo processing: MIMO and linear precoding iterative detection have been detailed to illustrate the gains that bi-directional message passing can offer. An original method, called genieinitialised iterative processing (GIIP) has been proposed to evaluate the performance of iterative processing. This method appears to give a tight lower bound of the maximum likelihood performance for any signal to noise ratio and thus can be viewed as a powerful tool to assess the quality of communication systems involving iterative receivers.

\section{REFERENCES}

[1] C. Berrou, A. Glavieux and P. Thitimajshima, "Near Shannon limit error-correcting coding and decoding: turbo-codes", Proc. of IEEE ICC '93, Geneva, pp. 1064-1070, May 1993.

[2] C. Douillard, A. Picard, P. Didier, M. Jézéquel, C. Berrou and A. Glavieux, "Iterative correction of intersymbol interference : turboequalization", European Trans. Telecommun., vol. 6, no. 5, Special issue on turbo decoding, pp. 507-511, Sept.-Oct. 1995.

[3] J. Hagenauer, "The turbo principle: tutorial introduction and state of the art", Proc. of the first Symposium on Turbo Codes \& related topics, Brest, pp. 1-11, Sept. 1997.

[4] R. G. Gallager, "Low-density parity-check codes", IRE Trans. Inform Theory, Vol. IT-8, pp. 21-28, Jan. 1962.

[5] D. J. C. MacKay, "Good error-correcting codes based on very sparse matrices", IEEE Trans. Inform. Theory, vol. 45, pp. 399-431, March 1999.

[6] G. C. Clark, Jr. and J. B. Cain, Error-Correction Coding for Digital Communications. New York: Plenum, 1981.

[7] DVB, "Digital Video Broadcasting", ETSI EN 301 210-v1.1.11999-03.

[8] P. Thitimajshima, Les codes convolutifs récursifs systématiques et leur application à la concaténation parallèle (in French), Ph.D. thesis, no. 284, Université de Bretagne Occidentale, Brest, France, Dec. 1993.

[9] J. Hagenauer and P. Hoeher, "Concatenated Viterbi-decoding", Proc. of Int. Workshop on Inform. Theory, Gotland, Sweden, Aug-Sep. 1989.

[10] C. Berrou and A. Glavieux, Reflections on the Prize paper : "Near optimum error correcting coding and decoding: Turbo codes", IEEE IT Society Newsletter, Vol. 48, no. 2, June 1998.

[11] C. Weiß, C. Bettstetter and S. Riedel, "Code construction and decoding of parallel concatenated tail-biting codes", IEEE Trans. Inform. Theory, vol. 47 , no. 1 , pp. 366-386, Jan. 2001.

[12] Consultative Committee for Space Data Systems, "Recommendations for Space Data Systems. Telemetry Channel Coding", BLUE BOOK, May 1998.

[13] S. Crozier and P. Guinand, "Distance upper bounds and true minimum distance results for turbo codes with DRP interleavers", Proc. of the $3^{\text {rd }}$ Symposium on Turbo Codes \& related topics, Brest, pp. 169-172, Sept. 2003.

[14] C. Berrou, Y. Saouter, C. Douillard, S. Kerouédan and M. Jézéquel, "Designing good permutations for turbo codes: towards a single model", Proc. of ICC'04, Paris, June 2004.

[15] IEEE Std 802.16a, "IEEE standard for local and metropolitan area networks", 2003, available at http://standards.ieee.org/getieee802/download/802.16a-2003.pdf.

[16] C. Douillard and C. Berrou, "Turbo Codes with Rate- $m /(m+1)$ Constituent Convolutional Codes", IEEE Trans. Commun., vol. 53, no. 10, pp. 1630-1638, Oct. 2005.

[17] L.R. Bahl, J. Cocke, F. Jelinek and J. Raviv : "Optimal decoding of linear codes for minimizing symbol error rate", IEEE Trans. Inform. Theory, IT-20, pp. 248-287, Mar. 1974.
[18] 'CAS5093: Turbo encoder-decoder', Data sheet, COMATLAS, France, Nov. 1993.

[19] C. Berrou, "Error-correction coding method with at least two systematic convolutional codings in parallel, corresponding iterative decoding method, decoding module and decoder", patent 9105280 (France), 5,446,747 (USA).

[20] G. Battail, "Pondération des symboles décodés par l'algorithme de Viterbi", (in French), Ann. Télécommun., France, vol. 42, no. 1-2, pp. 3138, Jan. 1987.

[21] J. Hagenauer and P. Hoeher, "A Viterbi algorithm with soft-decision outputs and its applications", Proc. of Globecom'89, Dallas, Texas, pp. 47.11-47-17, Nov. 1989.

[22] P. Robertson, P. Hoeher and E. Villebrun, "Optimal and suboptimal maximum a posteriori algorithms suitable for turbo decoding", European Trans. Telecommun., vol. 8, pp. 119-125, March-Apr. 1997.

[23] C. Berrou, "The ten-year-old turbo codes are entering into service", IEEE Commun. Mag., pp. 110-116, Aug. 2003.

[24] 3GPP Technical Specification Group, Multiplexing and Channel Coding (FDD), TS 25.212 v2.0.0, June 1999.

[25] Z. Wang, Z. Chi and K. K. Parhi, "Area-efficient high-speed decoding schemes for turbo decoders", IEEE Trans. VLSI Systems, vol. 10, no. 6, pp. 902-912, Dec. 2002.

[26] C. E. Shannon, A Mathematical Theory of Communication, Bell System Technical Journal, vol. 27, July and October 1948.

[27] R. J. McEliece, D. J. C. MacKay and J.-F. Cheng, "Turbo decoding as an instance of Pearl's 'belief propagation' algorithm", IEEE Journal on Selected Areas in Commun., vol. 16, no. 2, pp. 140-152, Feb. 1998.

[28] DVB, "Interaction channel for satellite distribution systems," ETSI EN 301790 , V1.2.2, pp. 21-24, Dec. 2000.

[29] R. T. Derryberry, S. D. Gray, and D. M. Ionescu, "Transmit diversity in 3G CDMA systems", IEEE Commun. Magazine, vol. 40, issue 4, pp. 6875, Apr. 2002.

[30] V. Tarokh, H. Jafarkhani and A. R. Calderbank, "Space-time block coding for wireless communications: performance results", IEEE Journal on Selected Areas in Commun., vol. 17, no. 3, pp. 451-460, Mar. 1999.

[31] G. J. Foschini, "Layered space-time architecture for wireless communication in a fading enviroment when using multiple antennas", Bell Labs Tech. Journal, vol. 1, pp. 41-59, 1996.

[32] W. Su and X. G. Xia "Signal constellations for quasi-orthogonal spacetime block codes with full diversity", IEEE Trans. Inf. Theory, vol. 50, no. 10, pp. 2331-2347, Oct. 2004.

[33] A. Chindapol and J. A. Ritcey, "Design, analysis, and performance evaluation for BICM-ID with square QAM constellations in Rayleigh fading channels", IEEE Journal on Selected Areas in Commun., vol. 19, pp. 944-957, May 2001.

[34] A. M. Tonello, "Space-time bit-interleaved coded modulation with an iterative decoding strategy", Proc. of IEEE Veh. Technol. Conf., pp. 473-478, Sept. 2000.

[35] Z. Hong, B. L. Hughes, "Bit-interleaved space-time coded modulation with iterative decoding", IEEE Trans. On Wireless Commun., vol. 3, no. 6, pp. 1912-1917, Nov. 2004.

[36] O. Damen, A. Chkeif and J. C. Belfiore, " Lattice Code Decoder for Space-Time Code", IEEE Communications Letters, Vol. 4, No. 5, May 2000.

[37] S. Kaiser, "OFDM-Code Division Multiplexing in fading channels", IEEE Trans. On Commun., vol. 50, no. 8, pp. 1266-1273, Aug. 2002.

[38] J. Boutros, E. Viterbo, "Signal space diversity: a power and bandwidth efficient diversity technique for the Rayleigh fading channel", IEEE. Trans. Inform. Theory, vol. 44, no. 4, pp. 1453-1467, July 1998.

[39] X. Ma and G. B. Giannakis, "Complex field coded MIMO systems: performance, rate and trade-offs", Wireless Comm. Mob Computer, vol. 2, pp. 693-717, 2002.

[40] J. Le Masson, Systèmes de transmission avec précodage linéaire et traitement itératif. Application à l'OFDM et aux techniques MIMO, (in French), PhD. Thesis, Université de Bretagne Sud, Dec. 2005.

[41] R. Le Bidan, Turbo-equalization for bandwidth-efficient digital communications over frequency-selective channels, $\mathrm{PhD}$. Thesis, INSA de Rennes, 2003.

[42] S. Tüchler, A. C. Singer and R. Koetter, "Minimum mean square error equalization using a priori information", IEEE Trans. On Signal Proc., vol. 50, no. 3, pp.673-683, March 2002. 
[43] J. Le Masson, C. Langlais and C. Berrou, "Linear precoding with low complexity MMSE turbo-equalization and application to the wireless LAN system", Proc. of ICC'2005, vol. 4, pp. 2352-2356, May 2005.

[44] Z. Wang, S. Zhou and G. B. Giannakis, "Joint coding-precoding with low complexity turbo-decoding", IEEE Trans. Wireless Commun., vol. 3, no.3, pp. 832-842, May 2004.

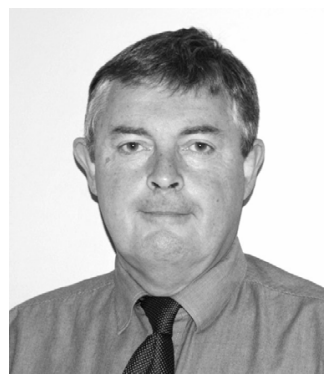

Claude Berrou was born in Penmarc'h, France, in 1951. In 1978, he joined the Ecole Nationale Supérieure des Télécommunications (ENST) de Bretagne, where he is currently a Professor in the Electronics Department. In the early 80 's, he started up the training and research activities in VLSI technology and design, to meet the growing demand from industry for microelectronics engineers. Some years later, Prof. Claude Berrou took an active interest in the field of algorithm/silicon interaction for digital communications. In collaboration with Prof. Alain Glavieux, he introduced the concept of probabilistic feedback into error correcting decoders and developed a new family of quasi-optimal error correction codes, that he nicknamed turbo codes. He also pioneered the extension of the turbo principle to joint detection and decoding processing, known today as turbo-detection and turbo-equalization. His current research topics, besides algorithm/silicon interaction, are electronics and digital communications at large, error correction codes, turbo codes and iterative processing, soft-in/soft-out (probabilistic) decoders, etc. He is the author and/or co-author of 8 registered patents and about 60 publications in the field of digital communications and electronics. He has received several distinctions, amongst which the 1997 SEE Médaille Ampère, the 1998 IEEE Information Theory Golden Jubilee Award for Technological Innovation, the 2003 IEEE Richard W. Hamming medal, the 2003 Grand Prix France Télécom de l'Académie des sciences and the 2005 Marconi Prize.

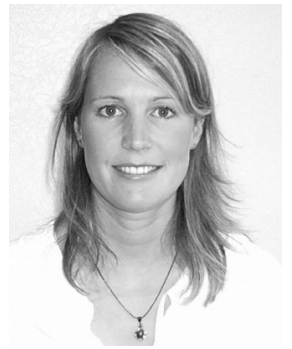

Charlotte Langlais was born in Le Mans, France, in 1976. She received the M.Sc. degree in electrical engineering in 1999 and the Ph.D. degree in electrical engineering in 2002, from the Institut National des Sciences Appliquées (INSA), Rennes, France. Since November 2002, she has been an Associate Professor at the Electronics Department, Ecole Nationale Supérieure des Télécommunications (ENST) de Bretagne, Brest, France. Her research interests include iterative processing for wireless communications, MIMO systems and multiuser communications.

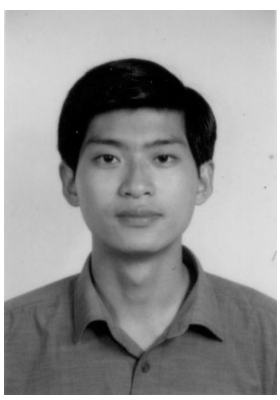

Yi Yu received his B.S. degree in computer engineering from the South-Central University for Ethnic Communities, China, in 2001 and his M.S. degree in information and communication from the Chonbuk National University, Korea, in 2004. Since September 2004 he has been with the Electronics Department at the École Nationale Supérieure des Télécommunications (ENST) de Bretagne, Brest, France, where he is currently pursuing his $\mathrm{Ph} . \mathrm{D}$. degree in electrical engineering. His main research interests include turbo codes and iterative decoding, iterative detection and space-time signal processing. 